ÉGYPTE monde arabe

\section{Égypte/Monde arabe}

$8 \mid 2011$

Développement durable au Caire : une provocation ?

\title{
La gestion des déchets ménagers au Caire : les habitants en question
}

Household waste management in Cairo : from the residents' perspective

\section{Safaa Monqid}

\section{(2) OpenEdition}

12 Journals

Édition électronique

URL : https://journals.openedition.org/ema/3003

DOI : $10.4000 /$ ema.3003

ISSN : 2090-7273

Éditeur

CEDEJ - Centre d'études et de documentation économiques juridiques et sociales

Édition imprimée

Date de publication : 1 septembre 2011

Pagination : 85-105

ISBN : 978-2-905838-47-7

ISSN : $1110-5097$

Référence électronique

Safaa Monqid, "La gestion des déchets ménagers au Caire : les habitants en question », Égypte/ Monde arabe [En ligne], 8 | 2011, document 8, mis en ligne le 01 septembre 2012, consulté le 07 juillet 2022. URL : http://journals.openedition.org/ema/3003 ; DOI : https://doi.org/10.4000/ema.3003 


\section{SAFAa MonQID}

\section{RÉSUMÉ / ABSTRACT}

\section{LA GESTION DES DÉCHETS MÉNAGERS AU CAIRE : LES HABITANTS EN QUESTION}

La ville du Caire, comme la plupart des villes arabes, connait un processus d'urbanisation accéléré, la démographie est galopante et les bouleversements économiques, sociaux et politiques ont des incidences directes sur le volume des déchets urbains qui sont produits chaque jour et qui sont en constante augmentation. Les autorités compétentes ont de grandes difficultés à les contenir et à les éliminer, comme en témoigne le spectacle des détritus qui jonchent le bord des routes et l'amoncellement des déchets dans des décharges sauvages. Aujourd'hui, on arrive souvent à une situation de crise puisque les répercussions sur l'environnement et sur la santé des habitants sont de plus en plus graves. La gestion des déchets demeure l'un des maillons faibles de la gestion urbaine et des services urbains en Égypte. Ainsi, nous allons présenter, dans ce qui suit, un état des lieux de la gestion des déchets au Caire, nous attardons plus particulièrement sur la perception qu'ont les habitants de ce problème.

\section{HOUSEHOLD WASTE MANAGEMENT IN CAIRO: FROM THE RESIDENTS' PERSPECTIVE}

The city of Cairo, like most Arab cities, is experiencing a rapid process of urbanization, population is soaring and economic, social and political upheaval impacts directly on the volume of municipal waste which is produced every day and is constantly increasing. The competent authorities have great difficulty in containing and eliminating waste, as evidenced by the sight of trash that litters the roadsides and the accumulation of waste in garbage dumps. Today, this often happens in a crisis situation because the impact on the environment and the health of the inhabitants is becoming more serious. Waste management remains one of the weak links in urban management and urban services in Egypt. Thus, we will present, in what follows, an overview of waste management in Cairo, and we dwell more particularly on the perception of the inhabitants of this problem. 
SAFAA MONQID est sociologue et professeur d'arabe au lycée français du Caire. Après deux ans de post-doctorat CNRS au Cedej, elle y continue ses recherches en tant que chercheure associée. Elle est responsable de deux programmes de recherche : "la gestion des déchets dans les pays arabes » et «Femmes villes et gouvernance locale dans le monde arabe ».

SAFAA MONQID is a sociologist and professor of Arabic at the French Lycée School in Cairo. After two years of post-doctoral research CNRS at CEDEJ, she continues her work as a research associate. She is responsible for two research programs: "Waste Management in the Arab countries" and "Women, cities, and local governance in the Arab world." 


\section{LA GESTION DES DÉGHETS MÉNAGERS AU CAIRE : LES HABITANTS EN QUESTION}

\section{LA GESTION DES DÉCHETS AU CAIRE : ÉTAT DES LIEUX}

u Caire, considérée comme la première mégalopole du
monde arabe, avec une population estimée à plus de 17 mil-
lions d'habitants, le secteur de la gestion des déchets a été longtemps laissé pour compte. L'élimination des déchets est toujours considérée comme un gaspillage d'argent si l'on considère le coût des matériaux, le coût de leur élimination et du recyclage, d'où l'absence d'une politique nationale de ce secteur devenu politiquement et économiquement très sensible. Il a souvent été pris en charge par des structures de gestion traditionnelle et informelle : la communauté des zabbâlîn qui assurait la collecte et le tri des déchets depuis les années 1950 (Dollet S., 2003)¹. Un service était également assuré par les gouvernorats mais ce service est limité et le secteur rencontre plusieurs problèmes, nous citerons parmi eux :

- Les contraintes institutionnelles et juridiques, liées essentiellement à l'absence de stratégie claire, de cadre juridique et de prescriptions réglementaires définissant les modalités de gestion de ce secteur et les responsabilités de chacun des acteurs concernés, notamment en ce qui concerne les déchets industriels et médicaux.

- Les contraintes financières dues en particulier à l'insuffisance de moyens financiers mobilisés pour la gestion des déchets dans les gouvernorats. Cela se traduit par un nombre insuffisant de véhicules, de personnels, de conteneurs, mais aussi par l'absence de taxes et de redevances spécifiques à la gestion des déchets, ce qui rend difficile le développement d'un système de recouvrement des coûts.

1. Dollet S., Une communauté traditionnelle face à la modernité. Le cas des zabbâlîn du Caire, mémoire de DEA de science politique, (dir.) E. Picard, Université d'Aix-Marseille III, 2003. 
- Les contraintes techniques, liées essentiellement à l'inadaptation des moyens matériels mobilisés pour la collecte et le transport des déchets, à l'absence de prescriptions techniques et de normes de contrôle des différentes opérations du système de gestion des déchets (collecte, transport, transfert, traitement...), mais aussi aux méthodes de rejet des déchets qui ne sont pas aux normes. Sans oublier le problème des décharges sauvages, non contrôlées et souvent mal situées qui constituent un problème de santé publique. De plus, ces décharges sont souvent situées à proximité des zones d'habitat et des grandes villes pour réduire les frais de transport ce qui engendre des odeurs nauséabondes.

- Enfin les contraintes liées à l'incivilité des habitants et qui référent aux aspects de l'éducation et de la sensibilisation.

\section{Les évolutions et les réformes du secteur}

C'est au cours des années 1970 que la gestion des déchets est devenue en Égypte un enjeu important qui fédère l'intérêt de différents acteurs : ONG, bailleurs de fonds et instances politiques égyptiennes à la fois locales et nationales, surtout après l'inauguration de la nouvelle politique d'ouverture économique du Président Sadat. La Banque Mondiale a ainsi fait de la gestion des déchets solides (SWM) un des piliers, avec l'infrastructure et le transport, de son premier projet de développement urbain en Égypte et de son grand projet de développement urbain au Caire, considérés comme les deux premiers principaux prêts au pays ${ }^{2}$. En 1983, on assiste à la création de deux organes centraux au Caire et à Giza afin de centraliser la gestion des déchets : Le Cairo Cleanising and Beautification Authority (CCBA) et le Giza Cleanising and Beautification Authority (GCBA).

Ce n'est qu'à partir de la fin des années 1990 et du début de la décennie suivante qu'un vrai mouvement de réformes a été initié par le gouvernement, conscient des pressions sur l'environnement et sur la qualité du cadre de vie des populations, surtout dans les tissus urbains à forte densité de population. De nouveaux instruments juridiques et institutionnels ont été créés. Ainsi, en 1999 fut créé le comité national de gestion des déchets solides et la première stratégie nationale concernant ce secteur a vu le jour en juin 2000 et, avec elle, la privatisation

2. Voir le projet déchet initié par le Cedej sur ce thème et qui regroupe une équipe de chercheurs pluridisciplinaire. Pour plus d'information, voir la page dédiée à ce projet sur le site du Cedej : http://www.cedej-eg.org/spip. php?article66 
du service en faveur d'entreprises étrangères, sous forme de partenariat public-privé, ce qui a transformé en profondeur le système de gestion antérieur (Debout L., 2007) ${ }^{3}$.

Le but du gouvernement, à travers ce nouveau système de gestion urbaine, était un gain d'efficacité et une meilleure gestion de ce secteur et de ses problèmes, devant lesquels l'État se trouvait désarmé4. L'objectif était également de réduire au maximum les activités du secteur informel qui échappait au contrôle de l'État. Le contrôle de la ville et de ses espaces passe par le fait d'y assurer un service public adéquat et qui réponde aux attentes de la population. Ainsi, la ville d'Alexandrie fut la première à en faire l'expérience en signant un contrat avec la société française Onyx (Debout L., 2007).

\section{La gestion des déchets demeure une problématique nouvelle et un des défis majeurs pour les planificateurs}

On constate, que malgré les efforts déployés pour moderniser le secteur à travers les différentes réformes entreprises et même si le bilan sur le plan de l'amélioration du service semble être positif au Caire, il n'en demeure pas moins que le secteur des déchets reste confronté à plusieurs difficultés. La gestion des déchets n'en est qu'à ses débuts et le secteur reste peu développé. Les difficultés rencontrées sont dues essentiellement à l'insuffisance d'études préalables et de préparation en amont à ces réformes, ainsi qu'à l'absence d'études prospectives, en terme de densité de population, de tissu urbain, à même de favoriser la connaissance et la maîtrise des coûts, du financement et recouvrement des frais. Dans certains cas, les contrats sont inappropriés à la réalité du terrain, sans oublier les blocages politiques, institutionnels et sociaux qui entravent l'activité des entreprises comme l'existence des décrets municipaux interdisant, par exemple, le passage de tout véhicule dans certaines rues ou le passage de camions sur certains ponts (Debout L., 2007).

Le partenariat public-privé pose aussi parfois des problèmes. Là encore, des difficultés entravent l'action des gouvernorats, car l'administration n'a pas été suffisamment préparée au système de délégation

3. Debout L., 2007, La libéralisation/modernisation en Égypte. La possibilité d'une réforme de la gestion urbaine? Le cas pionnier de la privatisation de la collecte des ordures ménagères au Caire et son impact sur la gestion de la métropole, mémoire de Master II, (dir) C. Lefèvre, Institut Français d'Urbanisme, Lyon, $81 \mathrm{p}$.

4. À titre indicatif, selon le Cairo Cleaning and Beautification Agency, le Caire produirait par jour environ 12000 à 15000 tonnes de déchets par jour, en plus des 4000 tonnes des déchets issus des matériaux de construction. 
des services. En effet, les pouvoirs publics rencontrent des problèmes dans la gestion du partenariat avec le secteur privé par manque d'expérience et de culture partenariale. Ils ont des difficultés à gérer et à faire des contrats avec le privé : plusieurs incohérences et malentendus sur les objectifs des contrats ont été la source de conflits entre les deux parties. Pour les entreprises, il s'agit de contrats de service et pour les autorités ce sont des contrats de qualité et elles évaluent la " propreté » et non le service rendu (Debout L., 2007). Les entreprises étrangères ont eu également du mal à comprendre et à s'adapter à la réalité sociale et culturelle du pays, elles ont découvert une nouvelle façon de gérer les ordures, la collecte porte-à-porte à laquelle les habitants sont habitués et attachés. Elles ont été également confrontées à l'incivilité des habitants qui se traduit par le vol et l'appropriation des bacs à poubelle pour d'autres besoins, par l'absence de tri à la source...

Les pouvoirs publics font également face à des problèmes dans l'élaboration des mécanismes de contrôle des activités du secteur privé à cause de l'absence de dispositifs législatifs et opérationnels adéquats en terme de qualification, de moyens matériels et de logistique. On relève également la présence de problèmes institutionnels et juridiques qui persistent. Ils se traduisent par l'absence de cadre et d'instruments juridique et institutionnel opérationnels qui définissent les responsabilités des différents acteurs et l'absence de politique nationale fédératrice de gestion des déchets faisant l'objet de suivi et d'évaluation. La gestion demeure fragmentée à cause de la multiplication des acteurs qui travaillent d'une manière indifférenciée (entreprises privées, publiques, chiffonniers) et où les responsabilités des uns et des autres se confondent, ce qui rend le service inefficient. Il est nécessaire d'améliorer le cadre réglementaire, de réduire le nombre d'acteurs à travers la planification de ce secteur. Une autre difficulté, et non la moindre, réside dans les lacunes dans les modes d'exécution, la formation et dans les compétences requises pour une bonne gestion de ce secteur.

Le manque de moyens financiers et matériels et le manque d'infrastructures de base accompagnant ces réformes, comme les équipements de collecte et d'élimination, les centres de transfert..., est particulièrement criant et ceci constitue une difficulté supplémentaire. Les capacités institutionnelles pour gérer les déchets sont toujours insuffisantes et les investissements dans le secteur continuent à être faibles. Les moyens financiers sont insuffisants pour gérer le secteur d'une manière efficace, sans oublier le problème du secteur informel que l'État veut à tout prix évincer. D'autres lacunes demeurent, elles constituent des défis essentiels pour l'avenir. Elles consistent, entre autres, en l'absence d'une véritable politique de gestion des décharges. Les 


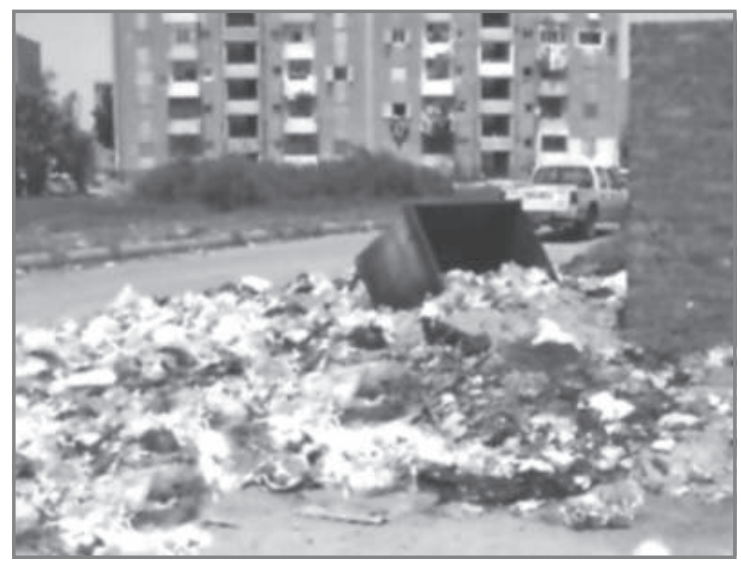

Figure 1- Photo S. Monqid, Madînat Nasr, avril 2009.

décharges sauvages

et non contrô-

lées constituent

un véritable pro-

blème de santé publique, d'autant qu'elles sont souvent situées à proximité des zones d'habitat, dans des sites sensibles, dévalorisant et paupérisant l'espace urbain. Il faut ajouter à cela les contraintes liées à l'éducation et à la sensibilisation des populations sur lesquels nous allons nous pencher de plus en plus en détail dans ce qui suit.

\section{HABITANTS ET DÉCHETS 5}

\section{Du point de vue des pouvoirs publics}

Selon les institutionnels, les contraintes liées à l'incivilité des habitants réduisent à plus de la moitié l'efficacité du service de la gestion des déchets. Les pouvoirs publics égyptiens considèrent la participation et la coopération des habitants comme étant insuffisantes. II n’y a pas de tri à la source, les ordures sont jetées d'une manière anarchique et n'importe où et non dans les lieux réservés à cela comme les bennes; les poubelles sont vandalisées et appropriées pour un usage personnel... Les responsables déplorent I'incivilité des habitants quant à la gestion de leurs ordures et leurs manquements aux règles de fonctionnement de la vie en collectivité, d'où la nécessité de campagnes

5. Une large enquête a été menée courant l'année 2009 auprès d'une cinquantaine d'habitants, dans les quartiers de Zamalek, Madînat Nasr, Maadi, le centre-ville, Shûbrâ, Qullalî, Boulaq, 'Izbit al-Nakhl, afin d'analyser leurs habitudes en matière de gestion des ordures ménagères, la perception qu'ils ont de l'action des pouvoirs publics, les compétences individuelles et I'action collective, surtout en cas de conflit (les supports de collecte des données qui ont été utilisés sont I'observation directe sur le terrain et les entretiens semi-directifs). 
de sensibilisation afin d'inciter les gens à évaluer leur propre rôle dans la propreté urbaine. Des conflits ont également éclaté lorsque le service est devenu payant, l'État estimant que le service a un coût auquel tout citoyen doit contribuer, quant au citoyen, il juge pour sa part que c'est un service public que le gouvernement doit prendre entièrement en charge.

L'étude de terrain révèle, en effet, l'existence de stratégie de résistances de la part des habitants qui restent attachés à la collecte de porte-à-porte, d'où les réticences de certains à aller jeter leurs ordures dans les bennes réservées car comme quelques habitants l'affirment: "Ce n'est pas dans notre culture ». Les gens ne sont pas habitués à porter eux-mêmes leurs ordures, cela est considéré comme dégradant, c'est une question d'honneur et de fierté, ils délèguent souvent cette tâche à I'aide-ménagère ou au bawâb. La préférence est à la collecte au porteà-porte par le chiffonnier qui vient récupérer les déchets à domicile. Les gens restent attachés au secteur informel, considéré comme plus fiable. "Le matin, je suis en costume-cravate et chemise blanche pour aller travailler et tu veux que je porte mon sac de poubelles et que je tourne dans le quartier jusqu'à ce que je trouve une benne, au nom de quelle loi, je ferai cela? Il faut d'abord que l'État prenne ses responsabilités. Même dans les pays les plus pauvres, tu trouves des bennes à ordures couvertes devant chaque porte, ils veulent (les pouvoirs publics) qu'on s'adapte, il faut alors qu'ils mettent les moyens nécessaires pour cela! ». Le chiffonnier fait partie du quotidien des Cairotes, il vient régulièrement dans les immeubles, pour la collecte ou pour la rémunération de son travail. Le porte-à-porte s'inscrit dans la tradition de service à domicile auquel sont attachés les Égyptiens. "Il y a aussi l'habitude, on aime que le zabbâl passe à la maison, on n'aime pas bouger. La compagnie ne collecte pas en porte à porte, ils ne viennent pas à la maison. On aime être servi, c'est une certaine culture ». L'aspect pratique est aussi mis en avant, comme le fait de ne pas descendre soi-même les ordures, surtout quand l'immeuble ne possède pas d'ascenseur.

Le tri collectif n'est pas non plus appliqué par la population. Seul le verre, qui est réutilisé, le pain rassis, parfois le papier sont triés. Le pain est soit revendu, soit donné comme nourriture aux oiseaux et aux poules, mais, en général, on ne le jette pas à cause de son caractère sacré. Il est considéré comme un don de Dieu (il est la base de l'alimentation). Les habitants, qu'ils appartiennent aux quartiers riches ou pauvres, trouvent la pratique du tri difficile à appliquer dans le contexte égyptien. Leur premier argument est que c'est une tâche qui revient prioritairement au zabbâl, c'est son gagne-pain. Les femmes évoquent aussi des questions d'ordre pratique comme le manque de place dans le foyer et le manque de sacs nécessaires à cette tâche. Le 


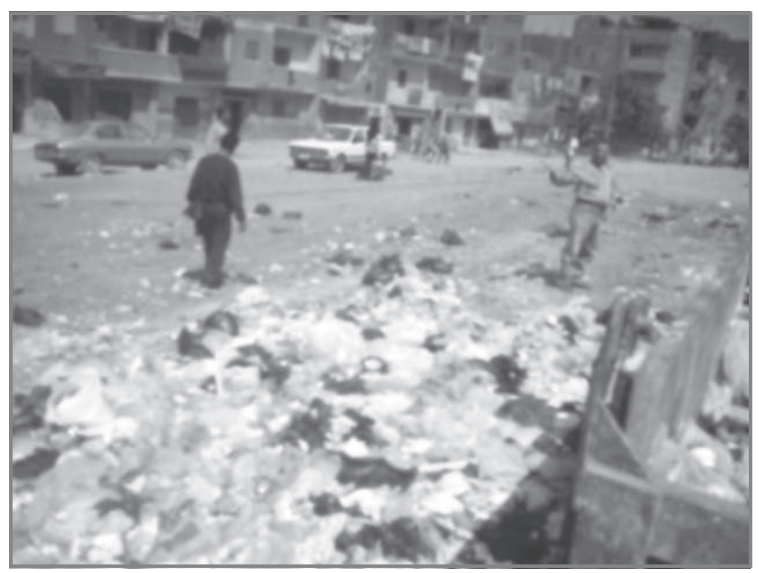

Figure 2- Photo S. Monqid, 'Izbit al-Nakhl, avril 2009. poids des modèles socioculturels est un facteur important à prendre en compte dans I'analyse du rapport des habitants à leurs déchets, car aucune action efficace pour dépasser ce problème ne peut être réalisée sans la prise en compte de cette dimension.

\section{Les habitants et leur perception du problème}

Les habitants pointent du doigt les lacunes de l'État dans ce domaine. Les deux camps se rejettent la responsabilité et on assiste, en reprenant les termes de $M$. Jolé, à " un jeu de dénonciation réciproque » (Jolé M., 1989) 6 . Pour eux, la qualité du service "laisse à désirer »; ils considèrent la gestion inefficace et le service anarchique ; ils déplorent I'insuffisance, voire l'absence de la conteneurisation et l'inadéquation des récipients de présentation des déchets, ce qui est à l'origine des décharges sauvages autour des bennes ou dans les terrains vagues ou entourés de murs aveugles, dans les parkings également, faute de lieu adéquat pour y jeter les ordures... Les habitants des quartiers démunis déplorent surtout l'inégalité du service entre les quartiers riches et les leurs, à savoir les quartiers pauvres du Caire. On assiste à une rupture de confiance entre les habitants et l'administration, considérée comme pas sérieuse, avec des fonctionnaires inaptes et corrompus, inefficaces et incompétents, comme le prouve d'ailleurs, aux yeux des habitants, le spectacle des déchets dans les rues. Ils exigent une administration plus responsable et plus attentive à leurs besoins, une administration prête à les écouter, mais aussi à les intégrer dans les prises de décision et dans les différents projets environnementaux, pour pallier à la fragmentation et à la participation limitée de la société civile à la formulation du politique et pour une meilleure cohésion urbaine.

6. Jolé M., 1989 « Le déchet ou l'autre côté de la limite », ndeg. spécial, Maghreb-Machrek, Espace et société dans le Monde Arabe, janvier. 
Les entretiens menés dans la ville du Caire montent l'insatisfaction des habitants vis-à-vis de la propreté de leurs quartiers et des espaces publics d'une manière générale. En effet, lorsqu'on demande aux gens de procéder à un classement des quartiers de la ville du plus propre au plus sale, seuls quelques quartiers sont considérés comme étant bien entretenus. Le quartier de Maadi vient en tête du classement puisqu'il abrite une importante population étrangère. D'autres quartiers résidentiels aisés suivent comme Zamalek, Héliopolis, Madînat Nasr, Muhandissîn, qui ont pour réputation d'accueillir " des gens importants », en reprenant les termes de nos interlocuteurs, d'où la régularité de leur entretien; puis Garden City car il contient des ambassades, sans oublier les différents compounds comme Mirage, Rihâb, Madînatî qui sont gérés d'une manière privée...; puis viennent les villes nouvelles comme la ville du 6 octobre qui sont moins denses en terme de population. Dans tous ces quartiers, le nettoyage et le ramassage des ordures seraient réguliers, peu de déchets jonchent les rues et on y trouve beaucoup d'espaces verts, contrairement aux quartiers populaires pauvres. Viennent par la suite des quartiers comme le centre-ville, " place Tahrîr » et Doqqi. Or, malgré leur propreté apparente, les habitants attestent du fait que plusieurs rues secondaires dans ces quartiers restent sales. Le centre-ville, considéré par ses habitants comme étant sale, est même pour beaucoup " dans un état catastrophique ", ce qui a poussé ses habitants aisés à le déserter vers d'autres quartiers considérés plus résidentiels et plus propres surtout vers les villes nouvelles comme celle du 6 Octobre. La présence continuelle de déchets dans le centreville serait due au fait que $c^{\prime}$ est une zone de fort passage, ce qui fait que même s'il a été nettoyé tôt dans la journée, les passants le salissent.

Quant aux quartiers réputés comme étant sales, on distingue des quartiers comme Imbaba, Boulaq, Bachtîl, el Qullalî, Chûbrâ alkhîma... Toutes les zones surpeuplées sont réputées pour être plus sales et laissées pour compte par les pouvoirs publics. L'espace habité dans ces quartiers donne l'impression qu'il ne fait l'objet d'aucune attention. Les gens se plaignent de l'insalubrité de leur lieu d'habitat à cause de I'amoncellement des déchets qui amènent des problèmes comme les moustiques, les mouches et qui peuvent aussi provoquer des maladies des yeux, sans oublier les odeurs nauséabondes, les habitants ont conscience des dangers qui les guettent du fait de l'exposition aux déchets : "Dans ces quartiers, on est anesthésié par les odeurs, en plus l'état des rues laisse à désirer, elles sont affreuses et les trottoirs y sont cassés et sont très sales, les rues secondaires ne sont jamais nettoyées, et cela a des conséquences néfastes sur notre santé, mais qui s'en préoccupe !!». On y déplore le manque ou l'absence totale de bennes à ordures et, quand ces dernières existent, elles ne sont pas vidées régu- 
lièrement, ce qui pousse les gens à jeter les ordures à côté. Les habitants se plaignent du passage irrégulier des entreprises, mais aussi du chiffonnier, ce qui les pousse à se débarrasser eux-mêmes des ordures, " on jette les déchets dans un coin sous le pont, parfois on y trouve des animaux morts, des ânes qui restent pendant deux semaines... ». Pour eux, le service de la gestion des déchets est inefficient d'autant qu'ils payent doublement le service sur la facture d'électricité et directement aux chiffonniers (entre 2 à 10 livres $^{7}$ selon le standing du quartier) : "On paye sur la facture le service, mais les ordures ne sont pas ramassées. Depuis qu'ils ont ramené les entreprises, rien n'est assuré, même le chiffonnier qui était régulier et qui venait tous les jours n'est plus fiable comme avant. Que voulez-vous qu'on fasse, aller nous plaindre à ceux qui viennent ramener la facture d'électricité ? Ils nous disent que ce n'est pas de leur ressort et que le travail consiste à venir récupérer l'argent. Ils prennent 5 livres et ne ramassent pas les ordures ». Les décharges sauvages situées à proximité des lieux d'habitation sont également montrées du doigt.

Le gouvernement est accusé de négligence. Les habitants déplorent la démission de l'État qui doit surveiller davantage et offrir plus de moyens aux professionnels du secteur pour leur permettre de mener à bien leur travail. Les responsables de quartier doivent aussi agir et s'occuper de ce problème. L'essence du problème serait le contrôle car personne ne contrôle le travail des entreprises et lorsque les employés viennent récupérer les poubelles, ils se contentent de ramasser les ordures faciles à récupérer et laissent le reste, sans que personne ne soit inquiété. Les habitants sont en colère à cause de l'inconfort et l'agacement dus aux ordures, les entreprises de nettoyage sont de plus en plus montrées du doigt par les Cairotes. Tout ceci n'est pas sans conséquence sur le sens civique et rend ainsi l'espace public, espace permissif de rejet, espace négligé et délaissé au profit de l'espace privé qui lui focalise toute l'attention. Les habitants reconnaissent que le problème est surtout un problème d'éducation et qu'une grande responsabilité incombe aux citoyens qui doivent modifier leurs comportements si I'État aussi fait le nécessaire : "Les gens ne sont pas civilisés, les entreprises n'assurent pas le suivi et l'État fait la sourde oreille, il faut qu'il contrôle et qu'il applique les sanctions et qu'il craigne Dieu ». Dans les quartiers populaires, ce sont souvent les nouveaux-arrivants, considérés comme n'ayant aucun ancrage et lien affectif avec le quartier de vie, qui sont accusés d'inculture urbaine et de rejets anarchiques dans le quartier.

7. Entre 24 centimes et 1,20 euro. 
Les habitants réclament le droit à une ville propre, à plus de propreté urbaine et $d$ 'hygiène pour le bien collectif, surtout les habitants des quartiers défavorisés où les passants sont obligés de se boucher le nez devant les tas d'ordures se trouvant sur leur passage. Mais, devant la démission de l'État, on note la prise en charge par les habitants de la propreté des espaces limitrophes, par des travaux de nettoyage et d'embellissement même si cela reste limité par rapport à ce qu'on a pu observer au Maroc où on constate une réelle appropriation des espaces limitrophes par les habitants, surtout les femmes (MonQID S., $2006)^{8}$. Il faut noter la multiplication des acteurs engagés et/ou concernés par le problème des déchets (médias, associations de défense de l'environnement, les habitants...), ce qui est révélateur d'une représentation commune de la crise engendrée par l'accumulation des déchets. Les habitants se substituent à l'État et ne restent pas passifs, ils se mobilisent, interviennent dans I'enlèvement des ordures soit individuellement soit collectivement en lançant des campagnes de propreté, des programmes de sensibilisation ou en formant des mouvements ou des associations ${ }^{9}$. Leur moyen d'action, $\mathrm{c}^{\prime}$ est leur volonté de rendre leurs rues propres. Les habitants ont pris conscience que $c^{\prime}$ est par l'engagement et la mobilisation qu'il faut envisager les solutions aux problèmes des déchets et que l'État seul ne peut tout faire. C'est ce que montre la campagne sur Facebook "Clean up Giza » qui a mobilisé plusieurs personnes, le 12 septembre 2009, pour nettoyer le quartier de Mohandessin, ce qui témoigne de l'amorce d'une conscience et d'une action collective. Nous présenterons, dans ce qui suit, l'entretien avec Dana Moussa passionaria du Caire propre. Cette jeune militante de 24 ans a réussi à mobiliser de nombreuses personnes pour ramasser les montagnes d'ordures accumulées lors des grèves des sociétés privées dans le gouvernorat de Giza, grâce à un appel qu'elle a lancé sur Facebook. Elle a su intéresser les médias à son combat et faire bouger les politiques. Toutefois, cette éco-citoyenne pense que c'est d'abord aux citoyens de se mobiliser pour améliorer leur cadre de vie.

8. MonQID S., Les femmes marocaines et la modernité urbaine, Presses Universitaires de Rennes, Rennes (parution prévue courant 2011) II s'agit d'un livre tiré de mon travail de thèse soutenu en 2006. Voir également sur l'appropriation de ces espaces le travail de Navez-Bouchanine F., Habiter la ville marocaine, Paris, L'Harmattan, 1997.

9. Sur le rôle des associations dans l'espace urbain égyptien voir BEN NefISSA S., Abd Al-Fattah N., Hanafi S., Milani C., (dir.), ONG et gouvernance dans le monde arabe, Paris/Le Caire, Karthala/Cedej, coll. Kalam, 2004. 


\section{CLEAN UP GIZA :}

\section{Entretien avec la responsable du mouvement Dana Moussa}

\section{Quelles sont les origines de votre militantisme?}

Je suis née et j'ai grandi au Caire. Nous sommes cinq dans ma famille, mes parents, ma sœur et mon frère. Nous sommes une famille de militants, mon père est encarté au Parti national et mon oncle est le chef du parti. Ils ont tous les deux été influencés par mon grandpère qui était un politicien et un membre actif dans le parti du Wafd, parti opposé au pouvoir, mais il est décédé avant que je naisse, alors que mon père venait à peine d'être diplômé de l'université. Avec mon oncle, ils ont dû prendre en charge leurs frères et sœurs, gérer l'entreprise familiale, tout en continuant leurs activités politiques. Le parti dans lequel ils militaient a connu, au temps de Nasser, des moments difficiles, c'est pour cette raison qu'ils travaillaient la plupart du temps en Algérie. Mon père a toujours joué un rôle dans la société et je pense être la seule dans ma famille à lui ressembler, il le dit souvent lors de nos discussions. Il ajoute: " lorsque tu mourras, le plus important ce n'est ce que tu as fait dans ta vie, mais ce que tu as laissé derrière toi ». $C^{\prime}$ est pour cela qu'il n'est pas question pour moi de me contenter d'une vie ordinaire, en allant le matin au travail et en rentrant le soir chez moi. Ainsi, chez moi, il y a toujours des débats politiques, nous discutons des problèmes du pays. Ma mère, quant à elle, apporte toujours un autre point de vue, car elle est originaire du Koweït.

Avec mon frère et ma sœur, nous avons étudié dans des écoles étrangères, britanniques puis américaines. Ces écoles nous ont appris comment exprimer nos opinions et nos amis étaient tous des étrangers. Il est vrai que nos études nous ont isolés du Caire et de ses problèmes, nous étions protégés par les murs de l'école et notre vie sociale se limitait à notre entourage. Nous apprenions les langues étrangères, nous écoutions une musique différente, nous lisions des ouvrages étrangers, nous pensions que le monde ressemblait à un petit village.

Après avoir obtenu mon baccalauréat, je suis allée aux États-Unis, à I'université à Boston. Une fois sur place, tout a changé pour moi, car j'ai vécu seule. En Égypte, je vivais en vase clos, avec les mêmes personnes, le même mode de pensée, j'avais ma famille, mes amis et si j'avais le moindre problème, je savais pouvoir compter sur eux. Aux États-Unis, les choses étaient plus compliquées, j'étais obligée de compter sur moi-même, d'aller me présenter, de me faire de nouveaux amis, de trouver un endroit pour vivre, de payer mes charges, de nettoyer ma maison et mon linge. Auparavant, c'était ma mère qui s'oc- 
cupait de tout cela. J'ai appris à réfléchir par moi-même, je me suis fait des amis Brésiliens, Turcs, Indiens, Jordaniens et d'ailleurs. J'ai remarqué qu'ils étaient tous ambitieux et qu'ils avaient tous d'autres activités en dehors de leurs études, des responsabilités qui les rendaient plus forts et qui les aidaient à affronter la vie. À titre d'exemple, l'équipe de football de mon université déblayait toutes les semaines la neige dans les rues de $6 \mathrm{~h}$ à $10 \mathrm{~h}$ du matin. Les membres de l'équipe étaient heureux de le faire. En Égypte, ce genre d'initiative n'existe pas.

J'ai commencé ainsi à participer à différentes activités et à suivre des modules supplémentaires à l'université. J'ai ainsi suivi un cours sur l'entreprenariat qui m'a été très utile par la suite. Je m'étais inscrite à plusieurs activités extrascolaires, car je n'aimais pas rester chez moi à ne rien faire. J'étais responsable, pendant trois années d'un camp pour enfants, "Graines de la paix », qui accueillait des enfants originaires de Palestine, d'Israël, d'Égypte, de Jordanie et d'Arabie Saoudite. On m'avait aussi choisi aussi pour aller à Den Haag, en Hollande, où se trouve le tribunal international, j'ai aussi participé à une rencontre à Harvard pour I'Union des peuples, cela a été formateur pour moi, car je m'intéressais déjà beaucoup à la politique et à l'économie.

Après avoir terminé mes études universitaires, j'ai décidé de rentrer dans mon pays, car j'aime mon pays et mes proches et parce qu'il y a beaucoup d'opportunités à saisir dans les pays en voie de développement comme l'Égypte. Certains pensent que nous souffrons de la famine et que nous sommes très pauvres, mais, au contraire, nous avons ici un potentiel énorme et tout est encore à faire. J'ai vu tellement de choses se faire aux États-Unis, que je me suis dit que je pourrais les réaliser aussi en Égypte. Ainsi, j'ai commencé à travailler chez Endeavor, une société à but non lucratif. La maison mère se trouve aux États-Unis, mais ils ont beaucoup de projets de développement en Amérique latine, en Jordanie, en Turquie, en Égypte et dans beaucoup d'autres pays. Travailler avec eux m'a permis d'élargir mon cercle de connaissance et de créer des réseaux. Notre mission était l'aide aux petites entreprises, nous nous attachions à établir un partenariat avec de grands hommes affaires pour les conseiller et les soutenir.

\section{Quand est né votre intérêt pour l'environnement?}

Ça a commencé dès l'université. J'ai été sensibilisée à cette question, grâce à une professeure qui était responsable des questions relatives au climat aux Nations Unies. Elle nous parlait beaucoup du climat, des différents protocoles et des négociations. C'était ma première source 
d'information dans ce domaine et cela m'a poussée à réaliser un travail dirigé sur la pollution des eaux en Égypte. Un ami m'a aussi parlé de I'action d'Enrique Penelosa, le maire de la ville de Bosta en Colombie. Dans ce pays qui est aussi pauvre que l'Égypte, cet homme a changé la ville de fond en comble. Il a réorganisé la circulation, amélioré les transports, créé des espaces verts. Il a fait des choses extraordinaires dans un pays qui est pire que le nôtre, puisqu'ils ont des problèmes de violence, de drogue, de mafia, alors pourquoi ne pas faire la même chose ici en Égypte? Son expérience a été un succès, les gens ont même commencé à se rendre à leur travail en vélo, c'est une expérience qui m'a beaucoup touchée.

Quand je suis rentrée des États-Unis, je suis restée une année inactive. J'ai écrit de la poésie et j'ai beaucoup lu. J'ai commencé à lire sur l'Égypte, car dans mon enfance je n'étais pas vraiment en contact avec mon pays, je ne connaissais pas ses problèmes et l'école américaine où j'étais se contentait de nous enseigner l'histoire des pharaons, ce n'est qu'une fois à l'université que j'ai pris un cours sur le Proche-Orient et j'ai été très intéressée par la question. J'ai commencé à réfléchir aux différents problèmes qui touchent notre société, dont celui de l'environnement.

Je pense qu'il faut qu'on rende à la terre chaque chose qu'on lui prend. On ne peut utiliser les arbres sans en replanter. Notre planète est en train d'agoniser, cela fait des années qu'on l'exploite et elle attend de nous qu'on soit reconnaissant, que l'on fasse des choses en retour. Il faut qu'un équilibre se créé. II y a des gens qui croient au réchauffement climatique, d'autres non, mais franchement cela ne change rien pour moi. Quand je vois les problèmes d'expansion urbaine, la surpopulation, les déchets, la pollution..., je trouve que nous sommes en danger, sans même le réchauffement climatique, et nous sommes lassés, en tant qu'êtres humains, de vivre au quotidien tous ces problèmes, il faut agir.

\section{Comment est né le mouvement «Clean Up Giza»?}

De retour au Caire et avant de travailler pour Endeavour, je suis allée à l'Institut Souires, une institution caritative, pour un entretien d'embauche, pour travailler avec eux sur la thématique des chiffonniers. Travailler avec eux m'a donné envie de réaliser un documentaire sur le sujet, tant le sujet était passionnant mais, à l'époque, je ne pensais pas vraiment à faire du travail de terrain jusqu'au jour où il y a eu les manifestations des zabbâlîn (chiffonniers), devant le siège du Gouvernorat de Giza, en septembre 2009. 
Avant les manifestations, je savais, comme la plupart des gens, que les chiffonniers faisaient du porte-à-porte pour ramasser les ordures et que les gens les payaient pour cela. Ils se chargeaient ensuite d'enfouir ou de brûler les déchets. Je savais aussi qu'ils faisaient du recyclage dans le quartier du Muqattam et que les chiffonniers étaient laissés pour compte dans la société, qu'ils étaient méprisés et que leurs droits n'étaient pas respectés. À cette époque, il y avait aussi des grèves, des problèmes entre les sociétés privées et les gouvernorats qui accusaient les sociétés de ne pas faire leur travail correctement alors que les sociétés accusaient l'État de ne pas honorer ses engagements.

Au moment des grèves de septembre 2009, les déchets n'ont pas été ramassés pendant plus de deux semaines. Normalement, j'habite dans un endroit propre dans le quartier de Mohandessin, mais il y avait tellement de déchets que je ne pouvais plus accéder à ma rue en voiture. II y avait des montagnes de déchets, on se serait cru dans un des quartiers pauvres. J'étais très énervée et lorsque j'ai demandé à un propriétaire de magasin, dans ma rue, de ramasser les déchets pour que les gens puissent passer, il m'a répondu que c'était le travail du chiffonnier et qu'il n'était pas un chiffonnier. Cela m'a fait rire et j'ai vu qu'il réfléchissait mal, car personne ne pouvait entrer dans sa boutique à cause des déchets. Ce qui lui importait, c'était son prestige social, il considérait honteux de mettre la main dans les poubelles. Ce même jour, je me rendais chez ma grand-mère et, une fois arrivée chez elle, j'ai annoncé à tous mes proches que je pensais réaliser une action sur Facebook, à l'adresse des gens qui s'intéressent à l'environnement comme moi, en les invitant à venir ramasser les ordures. Ma grand-mère m'a dit que personne ne viendrait, mon oncle et ma tante $m^{\prime}$ ont répondu la même chose, ils ont ajouté que j'aurais en plus des ennuis avec la police. J'ai essayé de les persuader du contraire en arguant qu'aux États-Unis, des jeunes déneigent les rues toutes les semaines et qu'en Égypte, nous avons aussi une jeunesse volontaire et dynamique.

J'ai donc organisé cet événement qui a eu un écho positif parmi la population, des médias m'ont contactée pour des interviews. J'ai décidé d'en profiter pour donner plus d'ampleur à l'événement. Madame Yousriyya Lousa, une philanthrope, a proposé de nous fournir des voitures pour ramasser les déchets et elle en a parlé au Gouverneur qui m'a téléphoné deux jours plus tard pour m'offrir son aide et pour me proposer des camions du Gouvernorat. Il subissait tellement de pressions et il était sujet à tant de critiques qu'il voulait profiter de cet événement pour redorer son blason, c'était un acte politique. J'ai aussi été contactée par la police de l'État qui m'a demandé ce que nous allions mettre comme vêtement. Ils nous ont déconseillé de mettre des vêtements d'une même couleur pour que cela ne ressemble pas à une manifestation. Le lieu de rendez-vous 


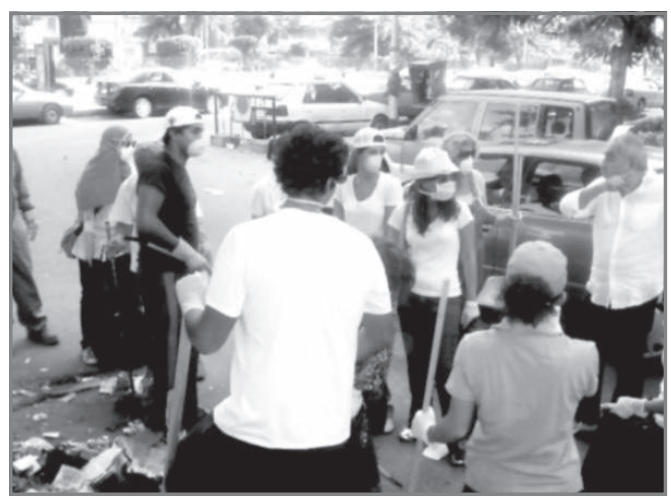

Figure 3- Mohandessin - photo, «Clean Up Giza », www.cleanupgiza.com/gallery.html des volontaires était fixé à I'Avenue de la Ligue arabe, dans le quartier de Mohandessin mais, comme la police d'État n'aime pas les regroupements de plus de dix ou vingt personnes, nous avons été obligés de nous constituer en quatre sous-groupes et chaque groupe est parti d'une zone précise pour commencer le nettoyage.

Nous étions environ 120 personnes, de tous les âges et de tous les milieux sociaux, il y avait beaucoup de médias, le journal al-Ahrâm, al-Masrî al-Yawm, l'émission al bît bîtak, Le Caire d'aujourd'hui... J'ai soudain pris peur, car l'affaire était pour moi très simple et elle ne méritait pas un tel tapage médiatique. Le gouverneur a pris la parole pour annoncer que le Gouvernorat de Giza avait réglé ses contentieux avec les entreprises et que les grèves étaient terminées. Nous avons été étonnés de trouver propres les rues que nous avions prévu de nettoyer. Le gouvernement avait envoyé des gens pour nettoyer les rues principales, car il savait que les médias allaient être présents. Mais les rues secondaires étaient toujours pleines de déchets et nous avons commencé à les nettoyer.

Un des volontaires a trouvé, dans un sac, un enfant endormi en plein milieu des montagnes de déchets, cela nous a beaucoup affecté, on trouve malheureusement beaucoup de cas similaires dans le quartier d'Imbaba.

L'événement s'est déroulé sur deux jours. C'était pendant le mois de Ramadan et il faisait très chaud. On travaillait de 12 à 17 heures. Nous avons ramassé l'équivalent de 600 à 700 sacs-poubelle. Des gens qui n'étaient pas au courant de l'événement se sont arrêtés, avec leur voiture, quand ils nous ont vus et ils sont venus nous aider. D'autres nous prenaient en photo en nous disant qu'ils aimeraient que leurs enfants suivent notre exemple. Les gens qui nous ont aidés à nettoyer nous disaient qu'ils avaient l'habitude de jeter les choses par la fenêtre de leur voiture et qu'ils n'avaient jamais pensé à ramasser les déchets dans la rue. En nettoyant avec nous, ils ont culpabilisé, tant il y avait 


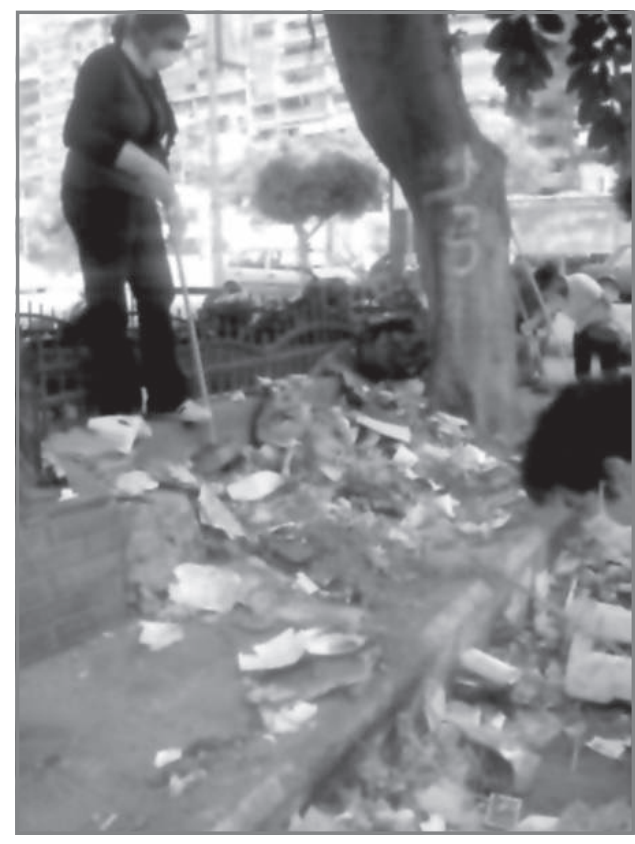

Figure 4- Mohandessin - photo, "Clean Up Giza », www.cleanupgiza.com/gallery. html des déchets. Dorénavant, jamais ils ne pourront refaire la même chose. Ces deux journées ont permis de faire connaître notre mouvement. L'événement est connu sous le nom de "Clean up Giza », mais le nom de la campagne en arabe est " châri' châri'na » (littéralement «La rue est notre rue»). Nous voulions un nom qui reflète notre mouvement et notre objectif qui consiste à pousser les gens à nettoyer leur quartier. Nous avons cherché un nom qui était facile à retenir pour les gens et qui reflète notre jeunesse et notre dynamisme. Nous avons nettoyé quatre zones : Mohandessin, de la place Lubnân à Mustafa Mahmud, la place Aswân, Jâmi'at al-duwal et la rive du Nil qui était une zone d'habitation presque transformée en décharge puisqu'on y déversait les déchets des rues principales. Ces deux jours de campagne ont été accompagnés d'un concert au centre culturel Sawî, c'était un moment festif, mais c'était en même temps l'occasion de parler des questions relatives à l'environnement.

La campagne ne nous a rien coûté puisque chacun a amené avec lui son matériel de nettoyage, ses gants, etc. Le deuxième jour, une société nous a fait don du matériel, mais on le lui a rendu ensuite. Nous ne nous sommes pas uniquement limités au ramassage des ordures, nous avons aussi planté des arbres. L'écho de cette expérience a été positif puisque plusieurs autres groupes se sont constitués par la suite pour nettoyer d'autres quartiers du Caire. Ils sont venus me demander des conseils sur les démarches à suivre et sur la nature des activités qu'ils pourraient mener et je les ai conseillés. Mais, j'ai remarqué que dès que le problème des grèves a été résolu, les gens n'étaient plus aussi motivés. Toutefois, l'essentiel est qu'on ait montré, par l'exemple, la volonté et le dynamisme de notre jeunesse. 


\section{Quel avenir pour ce mouvement?}

Tous les membres du mouvement se rencontrent au club pour discuter des différents problèmes et pour échanger sur les différentes questions d'ordre juridique économique, des questions de sensibilisation ou des activités à venir. Notre groupe n'est pas figé, les gens peuvent se joindre à nous à tout moment, d'autres partent. Ces rencontres sont plus importantes pour moi que l'action sur le terrain, car elles nous permettent de réfléchir à ce qui pourrait être fait et à la manière de procéder.

L'organisation de ces journées a été positive, mais cela n'est pas suffisant. J'ai envie de créer un site internet. II prendra la forme de cartes des différentes régions du Caire pour permettre au citoyen de nous signaler les déchets accumulés dans une rue donnée, en nous indiquant l'adresse exacte. Cela nous permettra de repérer les lieux où les déchets sont accumulés de faire pression sur le gouvernement. Les médias vont également écrire sur le sujet avec des preuves à l'appui. Notre site nous permettra ainsi de sensibiliser les entreprises et le gouvernement au problème, il sera bientôt lancé. Nous voulons développer le même principe, mais par SMS. Tout cela nécessite de I'argent. Nous sommes aussi en train de faire des démarches pour nous enregistrer en tant qu'association pour qu'on puisse trouver les financements nécessaires à notre développement et je chercherai des sponsors.

Le deuxième projet, que nous voulons développer se fera sur 5 à 10 ans. Nous en avons discuté avec l'Union européenne. Il consistera en I'installation de 25000 bennes à ordures qui pourront recevoir environ sept tonnes de déchets chaque semaine. Ce projet coûtera environ 30 millions de livres, mais il permettra de mettre fin aux plaintes des habitants qui disent n'avoir nulle part où jeter leurs ordures. C'est un projet qui nécessite d'avoir des partenaires solides comme de grandes entreprises ainsi que des institutions gouvernementales. Nous ne travaillons pas encore avec d'autres associations, car nous ne sommes pas encore nous-mêmes une association. Nous avons participé, avec d'autres institutions, à des événements concernant l'environnement, sponsorisés par de grandes entreprises la société Timberland par exemple, ou Coca Cola qui a organisé une grande journée pour le nettoyage de 'ayn sukhna.

Mon objectif est que le projet devienne autonome, qu'il fonctionne par lui-même, que je le vois grandir et qu'il soit utile à la société sans qu'il ait de leader. C'est quelque chose que j'ai appris aux États-Unis, ils ont de grands mouvements dont on ne connaît jamais les dirigeants. Je veux aussi être un modèle pour les autres, pour qu'ils aient le sens de l'entreprenariat. II faut que les gens se prennent en charge et qu'ils prennent des initiatives. 
De plus en plus dans le monde, les gens s'intéressent à l'environnement, en Égypte aussi, même si les choses arrivent chez nous avec un peu de retard. L'essentiel c'est qu'elles arrivent et qu'on travaille tous ensemble pour le bien de tous. Il y a une organisation de femmes qui fait la même chose que nous dans le quartier de Maadi, il y a des initiatives individuelles qui montrent qu'il y a une conscience nouvelle pour ces sujets. Le journal al-Masrî al-Yawm, a entrepris de publier chaque jour une photo d'un endroit rempli de déchets dans la ville, afin sensibiliser l'opinion publique et surtout de mettre des pressions sur les politiques. Lors de notre journée de nettoyage, ils sont venus nous prendre en photo et ils nous ont présenté comme la campagne des jeunes du al-Masrî al-Yawm, mais tout le monde sait que c'est faux et que nous étions un mouvement autonome. al-Masrî al-Yawm a organisé, après nous, une journée de nettoyage et nous avons tenu à y participer pour montrer que nous sommes ouverts et que nous œuvrons uniquement pour le bien de la société.

Nous sommes encore dans une phase de formation, il faut que nous nous fassions un nom, que l'on sache ce que l'on fait, car les gens ici se fient beaucoup aux opinions des autres. Il faut comprendre la psychologie des Égyptiens pour pouvoir les pénétrer de l'intérieur, il faut aussi leur donner l'impression qu'ils sont les premiers, qu'ils sont les initiateurs d'un mouvement et les encourager à persévérer, car chez nous les gens aiment se mettre en avant. Notre bilan est positif alors que cela fait seulement un an que nous avons lancé notre première initiative.

Enfin pour résoudre le problème des déchets, il faut tenir compte du triangle gouvernement, sociétés privées et citoyen. II faut que le citoyen soit conscient de ses responsabilités, et le jour où il en sera conscient, il fera pression sur les autorités qui mettront à leur tour plus de pression sur les sociétés pour un service plus efficace. II faut un travail en collaboration, cessé de rejeter la responsabilité les uns sur les autres. Il faut que les gens aient le sens du contrôle sur ce qui se passe autour d'eux, si je vois par exemple que ma voisine jette les déchets dans la rue, il faut que je me donne le droit d'aller la réprimander ou même de porter une plainte contre elle. La situation n'évoluera qu'à travers la sensibilisation et la sanction. Le gouvernement doit aussi tenir compte des chiffonniers qui ont toujours travaillé dans ce secteur et se doit d'améliorer leurs salaires, au lieu de les laisser mendier comme c'est le cas aujourd'hui. Il faut une gestion complète de cette question. La première chose à faire est d'expliquer aux habitants la nature des problèmes, car ensuite ils pourront mieux aider à les résoudre. Ce n'est que comme cela qu'on peut trouver les bonnes solutions et qu'on peut motiver les gens et les lier à une cause, le monde entier fonctionne comme cela, pourquoi pas nous? 


\section{RÉFÉRENCES BIBLIOGRAPHIQUES :}

- Abdel Motaal D., 1997, "The Zabbalin Community of Muqattam. Reconstructing Development: Women at the Muqattam Settlement », Cairo Papers in Social Science. The American University in Cairo, Egypt, 19(4), p. 59-110.

- Barthel P-A., Monqid S., 2011, Le Caire Réinventer la ville, Collection "villes en mouvement», (éd.) Autrement, Paris.

- Ben Nefissa S., 2007, "Ça suffit ? Le haut et le "bas" du politique en Égypte ", Politique africaine, n 108, L'Égypte sous pression, p. 5-25.

- Ben Nefissa S., Abd Al-Fattah N., Hanafi S., Milani C., (dir.) 2004, ONG et gouvernance dans le monde arabe, Paris/Le Caire, Karthala/ Cedej.

- Debout L., 2007, La libéralisation/modernisation en Égypte. La possibilité d'une réforme de la gestion urbaine? Le cas pionnier de la privatisation de la collecte des ordures ménagères au Caire et son impact sur la gestion de la métropole, mémoire de Master II, (dir.) C. Lefèvre, Institut Français d'Urbanisme, Lyon.

- Dollet S., 2003, Une communauté traditionnelle face à la modernité. Le cas des zabbâlîn du Caire, mémoire de DEA de science politique, (dir.) E. Picard, Université d'Aix-Marseille III, 193 p.

- Fahmi W. S., 2005, "The impact of privatization of solid waste management on the Zabaleen garbage collectors of Cairo », Environment and Urbanization, 17(2), p. 155-170.

- Florin B., 2010, Réforme du système de gestion des déchets au Caire et reconfigurations des territoires professionnels des zabbâlîn (chiffonniers), Géocarrefour, Lyon, 16 p.

- Gicguelay F., Goursat C., 2009, Les déchets au Caire, situations et habitants, Rapport de fin de stage, Safaa Monqid (dir.), Cedej.

- Goffman J.-P., 1973, La mise en scène de la vie quotidienne, (éd.) de Minuit, Paris.

- Jolé M., 1989, " Le déchet ou l'autre côté de la limite », ndeg. spécial, Maghreb-Machrek, "Espace et société dans le Monde Arabe ", janvier.

- Monqid S., s.p.a Les femmes marocaines et la modernité urbaine, Presses Universitaires de Rennes, (à paraître en 2011).

- Navez-Bouchanine F., 1993, "Les Espaces publics des villes marocaines", Annales de la recherche urbaine, numéro spécial, Espaces publics en villes, Paris, p. 184-190.

- 1994, "Initiatives populaires et développement urbain », nº spécial Monde Arabe Maghreb/Machrek, "Villes, pouvoirs et sociétés », La Documentation Française, p. 56-68.

- 1997, Habiter la ville marocaine, Paris, L'Harmattan. 